\title{
A patient with severe respiratory failure caused by novel human coronavirus
}

\author{
H. Guberina $\cdot$ O. Witzke $\cdot$ J. Timm $\cdot$ \\ U. Dittmer · M. A. Müller · C. Drosten • \\ F. Bonin
}

Received: 3 June 2013/ Accepted: 15 July 2013/Published online: 31 July 2013

(C) Springer-Verlag Berlin Heidelberg 2013

\begin{abstract}
We report a case of a 45-year-old patient who developed severe acute respiratory distress syndrome accompanied by renal failure. An infection with a novel human coronavirus was confirmed and found to be the reason for rapidly progressive respiratory failure of our patient.
\end{abstract}

Keywords Human coronavirus · Novel coronavirus ·

Middle East respiratory syndrome coronavirus

(MERS-CoV) - Acute respiratory distress syndrome .

Secondary pneumonia

\section{Introduction}

Four different human coronaviruses $(\mathrm{HCoV})$ are circulating worldwide, causing mostly mild and self-limiting respiratory tract infection. The outbreak of the severe acute

H. Guberina $(\bowtie) \cdot$ O. Witzke

Department of Nephrology, Essen University Hospital,

University Duisburg-Essen, Hufelandstr. 55,

45122 Essen, Germany

e-mail: hana.guberina@uk-essen.de

J. Timm · U. Dittmer

Institute of Virology, Essen University Hospital,

University Duisburg-Essen, Essen, Germany

M. A. Müller · C. Drosten

Institute of Virology, University of Bonn Medical Centre,

Bonn, Germany

F. Bonin $(\bowtie)$

Department of Intensive Care Medicine, Ruhrlandklinik, West

German Lung Centre, Essen University Hospital, University

Duisburg-Essen, Tüschener Weg 40, 45239 Essen, Germany

e-mail: frank.bonin@ruhrlandklinik.uk-essen.de respiratory syndrome (SARS) epidemic in 2002/2003 has clearly demonstrated how coronaviruses newly introduced into the human population can rapidly become pandemic, with high rates of associated case fatality [1, 2]. In September 2012 a novel $\mathrm{HCoV}$ emerged in the Middle East with severe clinical presentation resembling that of SARS$\mathrm{CoV}$ [3-5]. The prototype isolate of this new virus has been termed HCoV-Erasmus Medical Center (HCoV-EMC) [3]. As of May 2013 the World Health Organization (WHO) adopted a new name for this virus: 'Middle East respiratory syndrome coronavirus' (MERS-CoV) [6].

We describe here the complete clinical course of a patient infected with MERS-CoV. Under the precautionary measures taken, this virus does not seem to have led to secondary infections [7]. The patient was first treated in a local hospital in Qatar before being transferred to a specialist lung centre in Essen, Germany.

\section{Case presentation}

A 45-year-old, previously healthy, Qatari male was admitted to a local hospital in Qatar with high-grade fever, shortness of breath, tachypnoea and tachycardia on 13 October 2012. Additional clinical symptoms included cough, malaise and pronounced tiredness. These symptoms had suddenly appeared 1 week prior to admission. The patient denied past history of chronic diseases. However, he reported a long-term smoking habit of two to three packs of cigarettes a day for the past 30 years. Pulmonary auscultation revealed diffuse predominantly right-sided crackles. This finding was completely in agreement with chest radiography (CXR) findings of diffuse bilateral pulmonary infiltration, most pronounced on the right lower lobe. A severe community-acquired pneumonia was 
suspected and treatment with ceftriaxone and oseltamivir commenced. Despite the antibiotic treatment patient's respiratory status deteriorated so that $24 \mathrm{~h}$ later he required intubation for mechanical ventilation. The antibiotic therapy was changed to broad-spectrum antibiotics including piperacillin/tazobactam and azithromycin. The course of oseltamivir was sustained. None of the microbiological and virological diagnostics initiated during that time yielded clues to any pathogen. Regardless of remedial measures high-grade fever persisted and the patient's condition failed to improve. In the following days his respiratory status deteriorated further and an acute kidney injury additionally complicated his clinical course.

With diagnosis of acute respiratory distress syndrome (ARDS) of unknown origin, the patient was transferred for further treatment to Essen, Germany, on the 24 October 2012 (day 11 after hospitalization). On admission to the intensive care unit (ICU) the patient was haemodynamically stable, but in a poor respiratory condition, presenting hypercapnia with respiratory acidosis [partial pressure oxygen in arterial blood $\left(\mathrm{PaO}_{2}\right) 70 \mathrm{mmHg}, \mathrm{PaCO}_{2}$ $55 \mathrm{mmHg}$ ] and requiring a high level of oxygen supply (fraction of inspired oxygen 40-50\%) as well as highpositive end expiratory pressure (PEEP) ventilation. The subsequent CXR revealed progressive deterioration with diffuse interstitial infiltrates, and the computed tomography (CT) scan confirmed patchy bilateral peripheral lesions (Fig. 1a-c). Initial laboratory testing revealed leukocytosis of $16.1 \mathrm{GI} / 1$ (reference range 4-9 GI/1; with $72.3 \%$ of neutrophils in the differential count) and elevated inflammatory parameters. Kidney function had returned to normal with a creatinine level of $0.8 \mathrm{mg} / \mathrm{dl}$. On admission to the ICU a bronchoalveolar lavage (BAL) was performed, and subsequent examination of the BAL cultures revealed the presence of a multidrug-resistant Pseudomonas aeruginosa (with resistance against penicillins, cephalosporins and fluoroquinolones and with a reduced susceptibility to carbapenems) and Staphylococcus epidermidis. The antibiotic treatment was switched to colistin, clarithrimycin, imipenem/cilastatin and vancomycin. Cytological examination of BAL fluid showed an elevated neutrophil count and the presence of fungal pathogens, most likely Aspergillus fumigatus. Antifungal therapy with voriconazole was started. One week later an eosinophilia (14.7\% in the differential count) and elevated serum immunoglobulin (Ig) E levels (4,390 IU/ml; reference range: 4.4-100 IU/ml) were noted, raising suspicion of an allergic bronchopulmonary aspergillosis as additional cause for patient's prolonged respiratory failure. A prednisolone treatment $(50 \mathrm{mg} /$ day $)$ was commenced for a period of 1 week and produced a prompt improvement of the patient's symptoms. The oxygen supply and PEEP were reduced gradually. Based on the findings of the the CRX and CT scans, his chest findings and oxygen status had improved. As the patient became more stable, ventilator weaning began and on 16 November the patient was extubated. By this time his right lung infiltrates had mostly resolved (Fig. 1d-f). The results of the test of his lung function were within the normal range, and he could walk without any breathlessness. The patient was discharged from hospital on the 29 November 2012, after 48 days of hospitalization.

Only few days after the patient's discharge, we were informed that his bronchial specimen, taken on the 17 October 2012 in Qatar, had been tested positive by realtime reverse-transcription (RT) PCR for the novel-HCoV. Prior to receiving this information, the treating physicians had not taken the possibility of MERS-CoV infection into account. The test results had been communicated to the WHO on 21 November 2012. The RT-PCR performed as described by Corman et al. [5, 8] on stored specimens originating from the initial BAL upon admission in Essen (day 11 after hospitalization) tested positive for MERSCoV-RNA targets. The RT-PCR of the patient's nasopharyngeal aspirate taken on 23 November 2012 was negative by the same assay. Attempts to isolate the virus from both samples were unsuccessful. An immunofluorescence assay (performed as described by Corman et. al. [5, 8]) was used to detect antibodies produced in response to MERS-CoVinfection. This serological testing confirmed an infection with MERS-CoV. The patient had a specific anti-MERSCoV-IgM-titre in week 3 of illness (IgM titre 1:1,000). In week 8 his illness a seroconversion occurred, and the $\operatorname{IgM}$ titre decreased to $1: 100$, while the $\operatorname{IgG}$ titre was $1: 10,000$.

\section{Discussion}

In the case presented here, the severe respiratory distress syndrome was caused by infection with MERS-CoV and complicated by secondary pneumonia with multidrugresistant $P$. aeruginosa. There was also suspicion of a concomitant allergic bronchopulmonary aspergillosis (ABPA). The diagnosis of $\mathrm{HCoV}$ infection remained elusive until the patient was discharged. Our patient presented with rapidly developing respiratory failure leading to a severe ARDS. After surviving the infection, he was discharged from the hospital without any remaining deficiencies of his lung function.

In September 2012 two cases of respiratory failure induced by a novel human $\mathrm{CoV}$ were reported by the WHO. By the end of March 2013 a total of 17 laboratoryconfirmed infections with the novel coronavirus were reported, including those leading to 11 deaths [9]. Like most of the other patients, our patient was a middle-aged male resident of the Arabian Peninsula. He denied any recent travel history to foreign countries. He further did not 

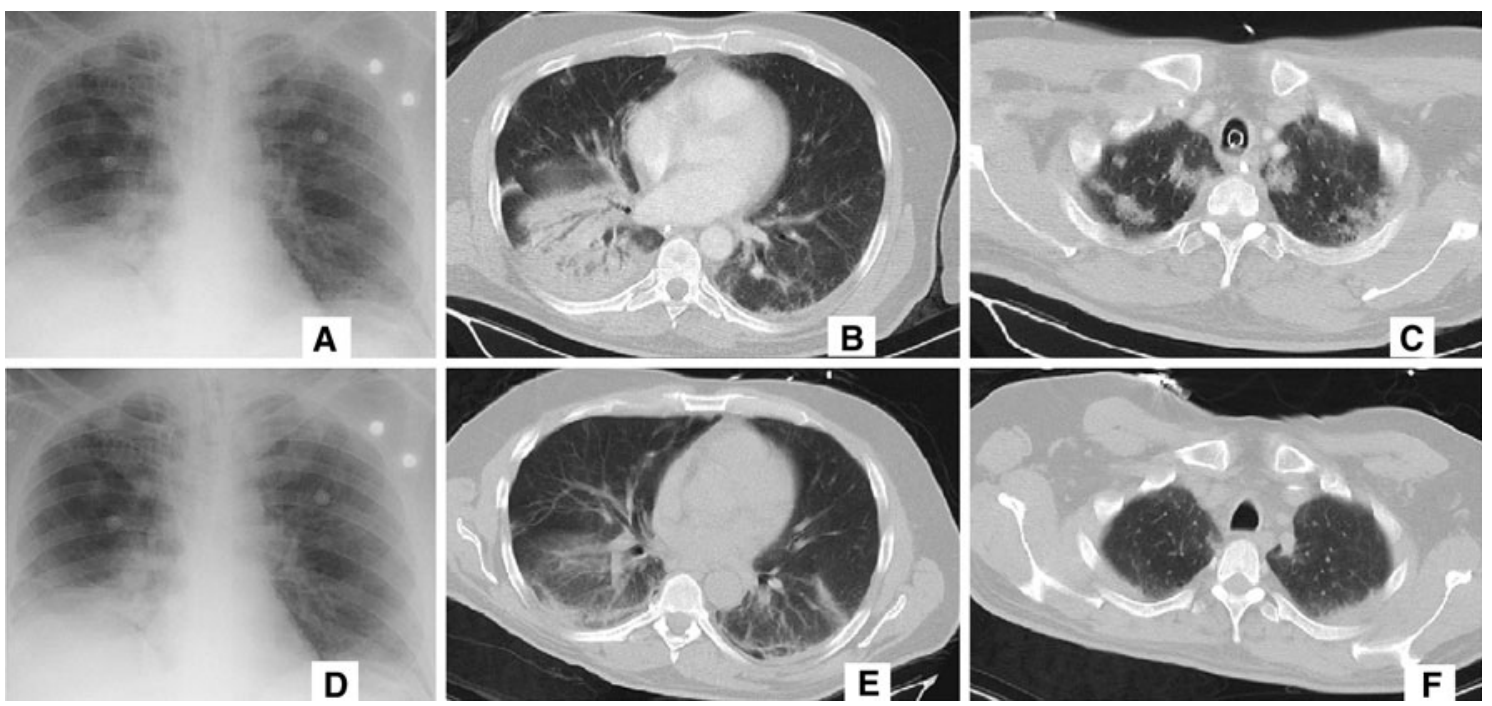

Fig. 1 Chest radiographs and computed tomography (CT) scans of the patient. a-c Chest radiography (a) and CT scans (b, c) obtained on October 24 show patchy bilateral peripheral lesions, mainly on the

remember being in contact with anyone with respiratory symptoms prior to onset of his illness.

To date, very little is known about the transmission pattern, severity and clinical impact of the novel human CoV. Ex vivo, MERS-CoV productively replicates in human bronchial and lung organ cultures [10], and it seems likely that MERS-CoV exhibits the same feature in vivo. A high concentration of the virus in the bronchial system of infected patients might lead to a high contagiousness, and procedures generating aerosols (such as airway suctions and bronchoscopies) are in particular considered to be a potential source for virus transmission. Such procedures were performed daily on our patient, whereas engaged healthcare workers only wore normal surgical masks without any further personal protective equipment (e.g. FP3-masks, protective eyewear, etc.). Due to the lack of information about patient's infection with MERS-CoV, only routine measures of isolation and protection had been taken during the whole course of the patient's treatment in the ICU. Given the nature of this virus, at that time the ICU healthcare staff was potentially at high risk of being infected with MERS-CoV. Together with public health authorities we therefore performed serological testing of all our workers and found no evidence of infection with MERS-CoV [7]. Furthermore, in the case of our patient there were no symptoms of MERS-CoV-infection within his family or circle of acquaintances. The fact that there have been only a limited number of reported and proven infections, may indicate a low potential of the virus to transmit readily between humans.

In the absence of an effective vaccine or specific antiviral therapeutic for the MERS-CoV infection, treatment right lower lobe. d-f Follow-up chest radiography (d) and CT scans $(\mathbf{e}, \mathbf{f})$ obtained on November 16 show improvement of aeration and less infiltration

remains supportive. The MERS-CoV is closely related to the SARS-CoV. In the treatment of SARS-CoV, no inhibitory effect was seen for oseltamivir (also used in treatment of our patient), while a possible beneficial effect of interferon, ribavirin or corticosteroids was observed [11]. Co-factors for the development of lung damage in patients with SARS infection are immune-mediated processes arising from overexuberant immune responses [12]. Consequently, corticosteroids are one backbone of immunomodulatory therapy in the treatment of SARS-CoV infections [13]. Their use leads to an alleged early improvement in terms of subsidence of fever, resolution of CXR infiltrates and better oxygenation [14]. The immunemodulating effect of corticosteroids may therefore also be beneficial in patients presenting an ARDS caused by MERS-CoV-infection. It was recently shown that interferon reduces the replication of MERS-CoV in human ex vivo lung cultures, thereby providing a stronger effect of immunomodulatory therapy for MERS-CoV-infection [10]. In our patient the respiratory condition did not improve significantly until treatment with corticosteroids was commenced.

In addition to the primary infection, we suspected a concomitant ABPA as an aggravating cause for our patient's prolonged respiratory failure. In addition to antimycotic therapy, an extended treatment with corticosteroids of up to 8 weeks is indicated to clear pulmonary infiltrates in patients with ABPA. In the case of our patient, the corticosteroids were administered only for 1 week. However, we cannot determine whether the corticosteroidinduced improvement of his respiratory status was achieved by treatment of the ABPA or of the MERS-CoV infection. 
It seems very probably that the clinical course of our patient was prolonged due to the severe lung injury, primary induced by the MERS-CoV-infection, and that this infection promoted the secondary pulmonary infection.

\section{Conclusion}

MERS-CoV has the potential to cause severe pneumonia in healthy humans. Even in immunocompetent patients, the infection can be a promoter of a secondary bacterial or fungal co-infection leading to a severe course of the disease. Immunomodulating substances such as corticosteroids or interferon may be beneficial in the treatment of virus-induced ARDS. Although the risk of transmission between humans seems to be low, appropriate infection control measures must be taken if MERS-CoV-infection is suspected. With regard to the severity of symptoms clinicians should consider MERS-CoV as a differential diagnosis in the case of patients with severe ARDS of unknown origin.

Conflict of interest None.

\section{References}

1. Drosten C, Gunther S, Preiser W, et al. Identification of a novel coronavirus in patients with severe acute respiratory syndrome. N Engl J Med. 2003;348:1967-76.

2. Peiris JS, Yuen KY, Osterhaus AD, Stohr K. The severe acute respiratory syndrome. N Engl J Med. 2003;349:2431-41.
3. Zaki AM, van Boheemen S, Bestebroer TM, Osterhaus AD, Fouchier RA. Isolation of a novel coronavirus from a man with pneumonia in Saudi Arabia. N Engl J Med. 2012;367:1814-20.

4. Bermingham A, Chand MA, Brown CS, et al. Severe respiratory illness caused by a novel coronavirus, in a patient transferred to the United Kingdom from the Middle East, September 2012. Euro Surveill. 2012;17:20290.

5. Corman VM, Eckerle I, Bleicker T, et al. Detection of a novel human coronavirus by real-time reverse-transcription polymerase chain reaction. Euro Surveill. 2012;17.

6. World Health Organization (WHO). Novel coronavirus infection-update (Middle East respiratory syndrome-coronavirus). Available at: http://www.who.int/csr/don/2013_05_23_ncov/en/. Accessed 23 May 2013.

7. Buchholz U, Muller M, Nitsche A, et al. Contact investigation of a case of human novel coronavirus infection treated in a German hospital, October-November 2012. Euro Surveill. 2013;18.

8. Corman VM, Muller MA, Costabel U, et al. Assays for laboratory confirmation of novel human coronavirus (hCoV-EMC) infections. Euro Surveill. 2012;17.

9. World Health Organization (WHO). Novel coronavirus infection — update. Available at: http://www.who.int/csr/don/2013 03_26/en/index.html. Accessed 26 Mar 2013.

10. Chan RW, Chan MC, Agnihothram S, et al. Tropism and innate immune responses of the novel human betacoronavirus lineage $\mathrm{C}$ virus in human ex vivo respiratory organ cultures. J Virol. 2013;87:6604-14.

11. Tan EL, Ooi EE, Lin CY, et al. Inhibition of SARS coronavirus infection in vitro with clinically approved antiviral drugs. Emerg Infect Dis. 2004;10:581-6.

12. Yoshikawa T, Hill TE, Yoshikawa N, et al. Dynamic innate immune responses of human bronchial epithelial cells to severe acute respiratory syndrome-associated coronavirus infection. PLoS One. 2010;5:e8729.

13. Chen RC, Tang XP, Tan SY, et al. Treatment of severe acute respiratory syndrome with glucosteroids: the Guangzhou experience. Chest. 2006;129:1441-52.

14. Tai DY. Pharmacologic treatment of SARS: current knowledge and recommendations. Ann Acad Med Singapore. 2007;36:438-43. 